\title{
“Crossing borders" SSIEM 2014 annual symposium in Innsbruck
}

\author{
Daniela Karall $^{1}$ - Sabine Scholl-Bürgi ${ }^{1}$. Johannes Zschocke ${ }^{2}$
}

Published online: 20 June 2015

(C) SSIEM 2015

This special issue brings together a series of articles resulting from the SSIEM 2014 Annual Symposium which took place on September 2-5, 2014, in Innsbruck Austria. We were pleased to welcome a record number of 2170 participants, including 71 students and trainees, from 73 different countries. There were 21 high profile plenary lectures on a wide range of topics from all fields of Metabolic Medicine. In addition there were 13 parallel sessions, with "Update" lectures and scientific free communications, as well as pre-opening specialist sessions on Nutrician \& Dietetics and Adult Metabolic Medicine. The Garrod Award 2015 was presented to Dr. Frédéric Sedel, Paris, in recognition of the article "Krabbe disease in adults: phenotypic and genotypic update from a series of 11 cases and a review" published in the JIMD in 2013 (Debs et al. 2013). The Komrower Lecture 2014 was held by Prof. Garry Brown, Oxford, on "A wide circle around lactic acid". The content of many plenary lectures is now also available as critically reviewed reference articles in this issue of the JIMD.

The general theme of the SSIEM Annual Symposium 2014 was "Crossing Borders". The people in Innsbruck know the challenges and excitement of climbing heights and crossing borders, and many participants crossed many borders to be in
Innsbruck for the symposium. The symposium featured a Public Lecture on "History and Health of the Iceman 'Ötzi" " by Dr. Angelika Fleckinger, South Tyrol Museum of Archaeology and Prof. Albert Zink, Institute for Mummies and the Iceman, Bolzano/Bozen, Italy, who talked about an ancient person who did not complete the crossing. Borders that separate and connect also play a central role in human metabolism, and new scientific heights can only be reached if we go beyond limits and work together. Metabolic medicine is evolving rapidly, and we hope that the meeting and now this issue of the JIMD will assist in reaching challenging new summits.

Conflict of interest None.

\section{Reference}

Debs R, Froissart R, Aubourg P, Papeix C, Douillard C, Degos B, Fontaine B, Audoin B, Lacour A, Said G, Vanier MT, Sedel F (2013) Krabbe disease in adults: phenotypic and genotypic update from a series of 11 cases and a review. J Inherit Metab Dis 36(5): $859-68$
Daniela Karall

Daniela.Karall@i-med.ac.at

1 Clinic for Pediatrics I, Inherited Metabolic Diseases, Medical University of Innsbruck, Anichstrasse 35, Innsbruck 6020, Austria

2 Division of Human Genetics, Medical University of Innsbruck, Innsbruck, Austria 